\title{
SPINOR: Visible and Infrared Spectro-Polarimetry at the National Solar Observatory
}

\author{
Hector Socas-Navarro, David Elmore, Anna Pietarila, Anthony \\ Darnell, Bruce W. Lites, \& Steven Tomczyk * \\ High Altitude Observatory, $\mathrm{NCAR}^{\dagger}$
}

Steven Hegwer

National Solar Observatory

Jun 28, 2005

\begin{abstract}
SPINOR is a new spectro-polarimeter that will serve as a facility instrument for the Dunn Solar Telescope at the National Solar Observatory. This instrument is capable of achromatic polarimetry over a very broad range of wavelengths, from 430 up to $1600 \mathrm{~nm}$, allowing for the simultaneous observation of several visible and infrared spectral regions with full Stokes polarimetry. Another key feature of the design is its flexibility to observe virtually any combination of spectral lines, limited only by practical considerations (e.g., the number of detectors available, space on the optical bench, etc).
\end{abstract}

Keywords: instrumentation: polarimeters, polarization, telescopes, Sun: magnetic fields, Sun: photosphere, Sun: chromosphere

\section{Introduction}

The new breed of spectro-polarimeters scheduled to begin operation in the next few years make the present a very exciting time for solar physics. Examples of these instruments are SOLIS (Keller 1998), SolarB (Lites et al. 2001), POLIS (Schmidt et al. 2003), Sunrise (Solanki et al. 2003), and the DLSP (Sankarasubramanian et al. 2003). All these instruments, however, are highly specialized and operate under fixed conditions, either with the aim of performing synoptic observations or to optimize for spatial resolution. While these aspects are very important for the future advance of solar physics, we feel that there is also a need for an "experiment-oriented" type of instrument. By this we mean an instrument that provides enough flexibility to implement more or less arbitrary optical arrangements at the observer's request. Such an instrument should allow researchers to observe any given spectral

\footnotetext{
* Visiting Astronomers, National Solar Observatory, operated by the Association of Universities for Research in Astronomy, Inc. (AURA), under cooperative agreement with the National Science Foundation.

$\dagger$ The National Center for Atmospheric Research (NCAR) is sponsored by the National Science Foundation
}

(c) 2018 Kluwer Academic Publishers. Printed in the Netherlands. 
line (or combinations of lines), either on the disk or off the limb at a diverse range of wavelengths. One should be able to modify parameters like spatial resolution, spectral dispersion, integration times, etc, in order to address a broad range of scientific problems. In some sense, operating an instrument of this kind is like setting up an experiment in a laboratory. Optical components and detector systems are arranged on an optical bench to investigate some specific problem.

For more than a decade, the highly successful Advanced Stokes Polarimeter (ASP, Elmore et al. 1992) has provided these capabilities at visible wavelengths. The Spectro-Polarimeter for Infrared and Optical Regions (SPINOR) will replace the ASP as the experimentoriented spectro-polarimeter on the Dunn Solar Telescope (DST) at the National Solar Observatory (NSO), Sunspot, NM, USA. SPINOR is currently under deveopment by the High Altitude Observatory and the National Solar Observatory. It has been conceived with flexibility as a top priority and intends to fill this gap in the next-generation solar instrumentation. The new instrument will replace and enhance the capabilities of the ASP.

Table I below provides some specifications for SPINOR. The most important enhancement over the ASP is the ability to observe simultaneously in the visible and the near infrared. Higher spatial resolution than was available over most of the life of the ASP is now possible thanks the the high-order adaptive optics (AO) system (Rimmele 2000) developed for the DST. Two different detectors will be available at commissioning time (scheduled for 2006): a PixelVision Pluto $652 \times 488$ thinned back-illuminated split frame transfer CCD, 60 frames per second readout rate (hereafter Pluto) and a Sarnoff CAM1M100SFT, 1024x512, thinned back-illuminated split frame transfer CCD, 100 frames per second readout rate. These will be fully dedicated for observations between $430 \mathrm{~nm}$ and $\sim 1 \mu \mathrm{m}$. The SPINOR optics allow for an even broader range of wavelengths, up to $1.6 \mu \mathrm{m}$, which will be accessible with an HAO Rockwell infrared camera that is shared with other HAO projects. The modular design of the instrument control allows for additional detectors to be attached to SPINOR using NSO's Virtual Camera interface.

This paper is organized as follows. Section 2 discusses the scientific motivations for the development of SPINOR. Section 3 presents a description of the polarimeter with some details on the new achromatic optics. The polarimetric calibration of the system consisting of the telescope and the polarimeter is explained in section 3.4. Some observations taken during two campaigns in June 2004 and May 2005 are presented in section 4 to illustrate the capabilities of the instrument. 
Finally, section 5 presents the main conclusions of our work and some future perspectives.

\section{Scientific Rationale}

The main scientific driver behind the development of SPINOR is to expand the present ASP magnetometric capabilities into the higher solar atmosphere. However, its infrared coverage is also of great relevance for the study of problems associated with photospheric magnetism. Below we summarize some of the major scientific problems for which SPINOR is particularly suited, which cannot be pursued using the ASP (or other existing instruments). However, being that this an experiment-oriented instrument designed for maximum versatility, its most important applications might be some that we cannot envision at this time.

\subsection{Photospheric magnetism}

Recent investigations of photospheric fields in the infrared often reveal a different picture from conventional visible observations. A remarkable example is the finding of supersonic flows in the penumbrae of sunspots by del Toro Iniesta et al. (2001) in data from the Tenerife Infrared Polarimeter (TIP). Such strong flows had never been observed in the visible, with the exception of the peculiar $\delta$-configuration sunspots (Martínez Pillet et al. 1994), and flows near pores (Lites et al. 2001).

Perhaps the most puzzling observations of photospheric fields in the infrared are those of the quiet sun, particularly outside the magnetic network (the region sometimes referred to as the photospheric internetwork). Visible observations indicate that most of the fields in this region are strong $(\sim 1.3 \mathrm{kG})$, but concentrated into very small areas ( $\sim 1 \%$ of the pixel; see Socas-Navarro and Sánchez Almeida 2002 and references therein). However, recent infrared observations (Lin 1995; Lin and Rimmele 1999; Khomenko et al. 2003) suggest that most of the fields are weak $(\sim 400 \mathrm{G})$ and diffuse. These seemingly contradictory results have sparked a controversy on the true nature of quiet sun fields. This issue is an important one, since our current understanding indicates that most of the solar magnetic flux (even at solar maximum) is located in the quiet sun outside of active regions, and it is likely that the evolution of this flux plays a role in the heating of the upper atmosphere. A possible solution to the observational contradiction has been proposed by Socas-Navarro (2003) and Socas-Navarro and Sánchez Almeida (2003). It turns out that the observations may be explained by 
a small-scale distribution of fields, beyond the spatial resolution of the observations, having intermixed weak and strong fields. Moreover, these authors showed that the actual sub-pixel distribution of the field can be inferred from simultaneous visible and infrared observations (like those from SPINOR).

The results of Socas-Navarro and Sánchez Almeida (2003) may be extrapolated to other physical scenarios in which different field strengths coexist within the resolution element of the observations. Another example where this strategy would be very useful is the investigation of sunspot penumbrae. The actual size, properties and origin of these filaments are still a subject of debate (Martínez Pillet 2000; Sánchez Almeida 2001; Martínez Pillet 2001). The ability to infer spatiallyunresolved distributions of the magnetic field from simultaneous visible and infrared observations would provide important clues on the structure of the penumbra.

Innovative new diagnostics of solar magnetic fields are emerging as a result of parallel theoretical and observational advances. In a recent effort to understand the anomalous polarization signals observed in some spectral lines, López Ariste et al. (2002) studied the hyperfine structure induced in the atomic energy levels by the nuclear spin, and its effects on the polarization transfer process. They demonstrated that the signature imprinted by the hyperfine structure on some spectral lines has an important potential for magnetic field diagnostics. Some of the most interesting lines lie in the wavelength domain between 800 and $1500 \mathrm{~nm}$. Examples are the Rb I D1 line at $794 \mathrm{~nm}$, which shows a combination of hyperfine structure and isotopic mix, or the Mn I lines at $870 \mathrm{~nm}$, and in the infrared at 1.29, 1.33 and $1.52 \mu \mathrm{m}$.

\subsection{Chromospheric magnetism}

A new global picture of solar magnetism is emerging from the seemingly disparate observational domains of photospheric small-scale magnetic fields and the diffuse, voluminous magnetic structure of the solar corona. The observation and interpretation techniques used for photospheric and coronal studies are markedly different. To further the development of this global view, we identify a key missing ingredient: an in-depth investigation of the interface layer, the chromosphere. Observational capability for chromospheric vector magnetic fields and the associated dynamics has been lacking because most interesting and/or useful lines lie outside the wavelength coverage range of most solar po-

larimeters (it should be noted, however, that successful investigations of prominence fields have been carried out recently; e.g. Casini and López Ariste 2003). Observable lines either form too low in the chromosphere 
(e.g., the Mg I $b$-lines) or their polarization transfer is still not well understood (e.g., $\mathrm{H}_{\alpha}$ ).

SPINOR would open new perspectives for chromospheric investigations with its ability to observe the full polarization state of the Ca II infrared triplet, around $854 \mathrm{~nm}$. These lines are the best candidates for chromospheric diagnostics, at least in the Zeeman regime, due to their relatively simple formation physics, their long wavelengths (which results in stronger Zeeman signals), and the valuable information they carry on the thermal and magnetic conditions of the higher atmosphere (Uitenbroek 1989; Socas-Navarro et al. 2000c; Socas-Navarro et al. 2000b). They are also sensitive to the Hanle effect, which provides complementary diagnostics on the weaker $(\sim 1 \mathrm{G})$ fields, and have been successfully modeled by Manso Sainz and Trujillo Bueno (2001).

Finally, the He I multiplet at $1083 \mathrm{~nm}$ is of great interest for chromospheric studies. This line is seen in emission in prominences and in absorption in filaments, with strong polarization signals arising from both Hanle and Zeeman effect. SPINOR would be able to provide full spectro-polarimetry at $1083 \mathrm{~nm}$, which implies the potential to investigate the magnetic and dynamic conditions of these structures. Other interesting coronal lines that may be accessible for observations are the two Fe XIII lines at $1074 \mathrm{~nm}$, although these may not be visible using a traditional solar telescope.

\section{Description of the instrument}

SPINOR is based on the design of the ASP, which uses a rotating waveplate as a modulator and a polarization beam splitter as a dualbeam analyzer. All of the polarization optical components, however, have been replaced by new achromatic ones. SPINOR utilizes the ASP calibration/modulation unit (cal/mod) at the exit port of the DST, high-order AO, and the Horizontal Spectrograph (HSG). Its optical components are distributed throughout the DST at the same locations as those for the ASP (Elmore et al. 1992). The entrance window can be covered with the achromatic linear polarizer array. Achromatic calibration and modulation optics reside in the calibration/modulation unit just above telescope Port 4 . The achromatic polarizing beam splitter is just behind the entrance slit of the horizontal spectrograph. Camera lenses, pre-filters, and high speed cameras specific to SPINOR are located within the HSG.

Since SPINOR operates over a much wider wavelength range compared to ASP, it was deemed necessary to allow SPINOR to use a selection of gratings. Depending upon the desired spectral line and 
Table I. Performance comparison between SPINOR and ASP

\begin{tabular}{lccc}
\hline Parameter & ASP & SPINOR \\
\hline Calibratable wavelength range (nm) & & $450-750$ & $430-1600$ \\
Field of view along slit (arc seconds) & 80 & 120 \\
Quantum efficiency & $430 \mathrm{~nm}$ & 0.01 & 0.72 \\
& $700 \mathrm{~nm}$ & 0.32 & 0.80 \\
& $1080 \mathrm{~nm}$ & 0.00 & $0.03,0.60^{*}$ \\
& $1600 \mathrm{~nm}$ & 0.00 & $0.60^{*}$ \\
& & & $*$ (with suitable IR camera) \\
\hline
\end{tabular}

spectral resolution, different gratings may be selected. Higher blaze angle gives higher spectral resolution for the same spatial sample size. Since SPINOR will operate with AO, its design favors a higher spatial resolution than the ASP. As a general-purpose research instrument, it cannot be optimized for a single line, so the SPINOR spatial resolution is not as high as that attainable by the new DLSP. These spectrograph issues lead typically to a spectrograph using a $40 \mu \mathrm{m}$ slit and $1000 \mathrm{~mm}$ camera lens. The observations reported in this paper were carried out using the 308.57 line/mm grating with a blaze angle of $52^{\circ}$.

Users can choose among several NSO gratings (see http://nsosp.nso.edu/dst/userman/instruments/ which combined with the other variables permit a wide range of resolutions and spectral ranges. As an example, for the Sarnoff camera, $630.2 \mathrm{~nm}, 50 \mu \mathrm{m}$ slit, $1000 \mathrm{~mm}$ camera lens focal length, 316 line $/ \mathrm{mm}$ grating, and 63 degree angle of incidence, resolving power is 170,000 , and spectrum length is $1.2 \mathrm{~nm}$.

\subsection{Specifications}

SPINOR has been conceived keeping versatility as the highest priority in order to allow for a broad range of potential applications. The project is being developed in a way that allows the ASP to remain operational until the new instrument is completed. Its most important features are:

- Achromatic optics from 430 to $1600 \mathrm{~nm}$ offer the capability of simultaneous observations at diverse wavelengths This extended range represents an important improvement over that of the ASP. Its potential scientific benefits have been discussed in more detail in section 2 .

- Detectors with higher quantum efficiency than those in ASP allowing for higher signal to noise observations. 
- Use of state-of-the-art components, electronic systems, computers, detectors and software. Deployed in 1991, ASP's technology is now 15 or more years old, and some of its systems are starting to fail. Both routine maintenance tasks and normal operation are compromised because many replacement parts are no longer available. The SPINOR system will be more stable and suffer significantly less downtime. Data products will also be easier to handle (e.g., using DVDs instead of magnetic tapes, simpler analysis procedures, etc), an improvement that will greatly facilitate the science and make the instrument more accessible to a broader user community.

- Open and modular optical design, with room on the optical bench to incorporate and/or replace components. SPINOR will be deployed at the DST as a set of instrument modules with "virtual" cameras, a concept that NSO has developed for the DLSP and other DST instrumentation. This will allow diverse and complex observations, combining SPINOR with other DST instruments. The control software will be fully customizable for a broad variety of observing modes (although several pre-defined modes will exist for frequently used configurations).

\subsection{Achromatic polarization optics}

Fig 3.2 shows the retardation as a function of wavelength for the SPINOR bicrystalline achromatic retarders (Meadowlark Optics, see Guimond and Elmore 2004). Plates of different retardations are obtained by varying the thicknesses of the quartz and sapphire parts (maintaining the thickness ratio). SPINOR uses a 1/4 wave retarder for polarization calibration, a 0.375 wave retarder for polarization modulation, and a 0.50 wave retarder to perform a coordinate transformation between the slit and the polarizing beam splitter. Crystals in the calibration retarder are broad-band anti-reflection (AR) coated and air spaced using epoxy with glass beads imbedded. One side of the $50 \mathrm{~mm}$ calibration polarizer uses $30 \mu \mathrm{m}$ beads, the other side $10 \mu$ beads. The $50 \mathrm{~mm}$ diameter polarization modulator consists of, in order, a broad band AR coating, sapphire, $\mathrm{CeF}_{3}$ coating, adhesive, quartz, and a broad band AR coating. The $25 \mathrm{~mm}$ diameter half-wave retarder is constructed like the modulator. The broad band AR coating (from ZC\&R) has a reflectance typically $<1.5 \%$ from $450 \mathrm{~nm}$ to $1600 \mathrm{~nm}$.

Fig 3.2 shows the theoretical polarimeter response matrix as a function of wavelength for SPINOR. This is calculated using the retardance design curves for the bicrystalline achromatic modulator and half-wave retarder behind the slit. 


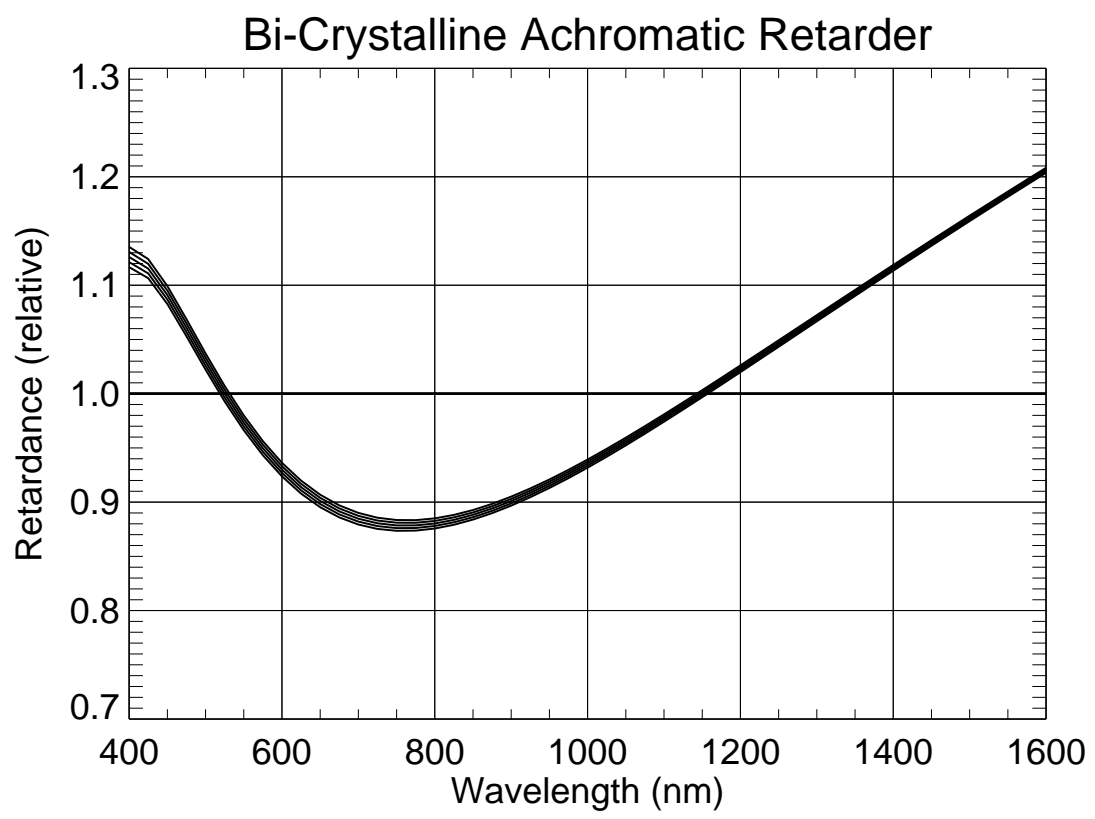

Figure 1. Design performance for the SPINOR retarders as a function of wavelength. There are two curves shown. The upper one is for $20 \mathrm{C}$ and the lower one for $30 \mathrm{C}$. These are shown for a one wave of retardance.

SPINOR uses wire grid polarizers (Versalight) for the telescope entrance window polarizers, calibration polarizer, and in the polarizing beam splitter. These polarizers transmit one orientation of linear polarization and reflect the orthogonal polarization. Versalight aluminum wire grid polarizers produce an extinction ratio of 400 at $450 \mathrm{~nm}$ and 4000 at $1650 \mathrm{~nm}$. There is a dip at $950 \mathrm{~nm}$ to just under 1000. The wire grid side of the polarizer can easily be damaged, therefore entrance window polairzers have the wire grid surface protected by a $\mathrm{MgF}_{2}$ coating. The calibration linear polarizer has custom broad band AR coatings optimized for the wire grid and glass surfaces. The polarizing beam splitter cube is a sandwich of two 90-degree prisms with the polarizer in between, cemented with an index matching adhesive.

\subsection{DeteCtors}

SPINOR uses the HSG at the DST, which sits on top of an optical bench and allows for great flexibility in the instrumental arrangement. The data presented here have been collected using a Rockwell TCM 8600, Pixel Vision Pluto, and the ASP TI TC245 cameras. SPINOR also has a dedicated Sarnoff CAM1M100. Any high frame rate camera that reads out upon an external strobe that is connected to a computer capable of 

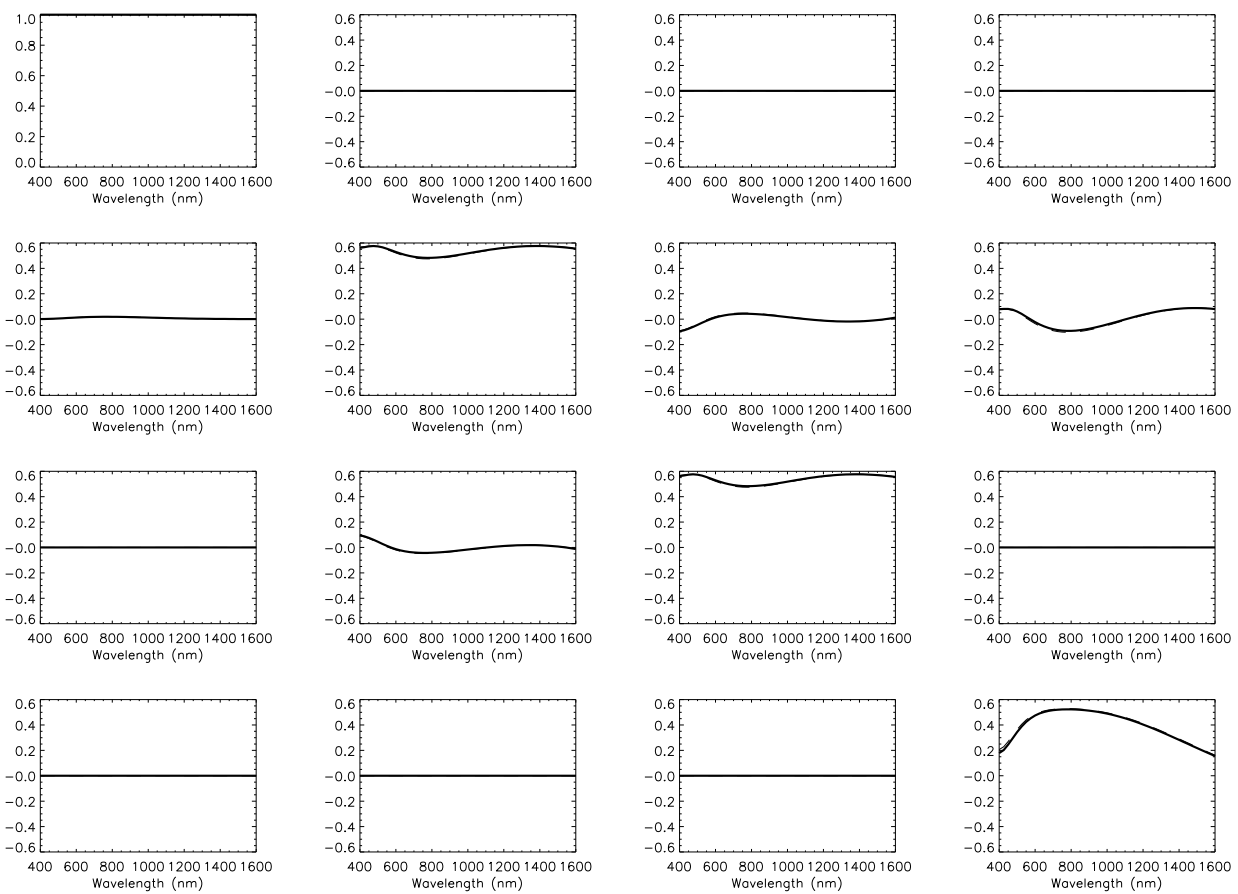

Figure 2. Theoretical polarimeter response matrix elements as a function of wavelength. Each plot represents a matrix element. The solid and dashed curves, which virtually overlap at the scale of the figure, represent different temperatures of 30 and 20 degrees Celsius, respectively.

storing the images according to the modulator status as received on a printer port can be connected to SPINOR. Camera computers receive modulator position information on their parallel ports, which is used to phase the camera reads to the nearest $1 / 16$ of the modulator rotation. The following table lists properties of current and possible SPINOR cameras. The SWIR camera listed is similar to cameras produced by Sensors Unlimited and Indigo.

Quantum efficiency values are vendor specifications and values in parenthesis are extrapolated. Frame rates with an asterisk can be increased with reduced field of view. Read noise values are measured except for the SWIR camera, which is a vendor specification.

Spatial pixel size and field of view vary depending upon the focal length of the spectrograph camera lens used. Figure 3.3 shows pixel spatial sample size and field of view for various cameras. This plot assumes the DST HSG with standard $3040 \mathrm{~mm}$ focal length collimator lens and the standard $\mathrm{f} / 36$ beam feeding the spectrograph, which produces a $133 \mu \mathrm{m} /$ arc-second plate scale at the HSG entrance slit. 
Table II. Quantum efficiency (as a function of wavelength), and other relevant parameters of various cameras for SPINOR.

\begin{tabular}{cccccc}
\hline $\begin{array}{c}\text { Wavelength } \\
(\mathrm{nm})\end{array}$ & $\begin{array}{c}\text { Sarnoff } \\
\text { CAM1M100 }\end{array}$ & $\begin{array}{c}\text { PixelVision } \\
\text { Pluto or BioXight }\end{array}$ & $\begin{array}{c}\text { ASP } \\
\text { TI TC245 }\end{array}$ & $\begin{array}{c}\text { Rockwell } \\
\text { TCM8600 }\end{array}$ & SWIR \\
\hline 450 & .73 & .73 & .20 & - & - \\
650 & .78 & .85 & .42 & - & - \\
850 & .59 & .46 & .30 & - & - \\
1100 & $(.1)$ & $(.05)$ & $(.02)$ & $>0.50$ & 0.65 \\
1600 & - & - & - & $>0.50$ & 0.70 \\
\hline Max Frame & & & & & 30 \\
Rate (fps) & $100^{*}$ & $50^{*}$ & 60 & & $>29^{*}$ \\
Read Noise & & & 50 & 70 & $\sim 300+$ \\
electrons $/ \mathrm{read}$ & 40 & 50 & $230 \times 512$ & $1024 \times 1024$ & $512 \times 640$ \\
Format & $512 \times 1024$ & $488 \times 652$ & $8.5 \times 19.75$ & $18 \times 18$ & $25 \times 25$ \\
Pixel size $(\mu \mathrm{m})$ & $16 \times 16$ & $12 \times 12$ & & & \\
\hline
\end{tabular}

Typical SPINOR slit widths are $50 \mu \mathrm{m}(0.375 ")$ or $80 \mu \mathrm{m}(0.60 ")$. In computing the field of view, pixel size, camera lens focal length, and the full field of view of the polarizing beam splitter have been considered. The polarizing beam splitter has a field of 145 " with a 30 " separation between the two beams.

\subsection{Calibration And Characterization}

The calibration procedure for SPINOR consists of the determination of the Mueller matrix of the telescope $(\mathbf{T})$ and the polarimeter reponse $(\mathbf{X})$. Any solar Stokes vector $\mathbf{s}_{\mathbf{i n}}$ is observed as $\mathbf{s}_{\mathbf{o u t}}$ (which is not necessarily a Stokes vector):

$$
\mathbf{s}_{\text {out }}=\mathbf{X T} \mathbf{s}_{\text {in }} .
$$

The polarimeter response matrix depends on the particular instrumental configuration and varies significantly between observing runs (sometimes even from one day to the next). For this reason, polarimeter calibration operations are done typically once a day, or even more often if changes are made to the instrument. Fortunately, calibration operations do not take too long ( $\sim 20$ minutes). A calibration linear polarizer and retarder are mounted following the telescope exit window. These are used to polarize the light beam in various known states. The known input vectors and the observed outputs are used in a leastsquares fitting procedure to determine the matrix elements of $\mathbf{X}$. This 


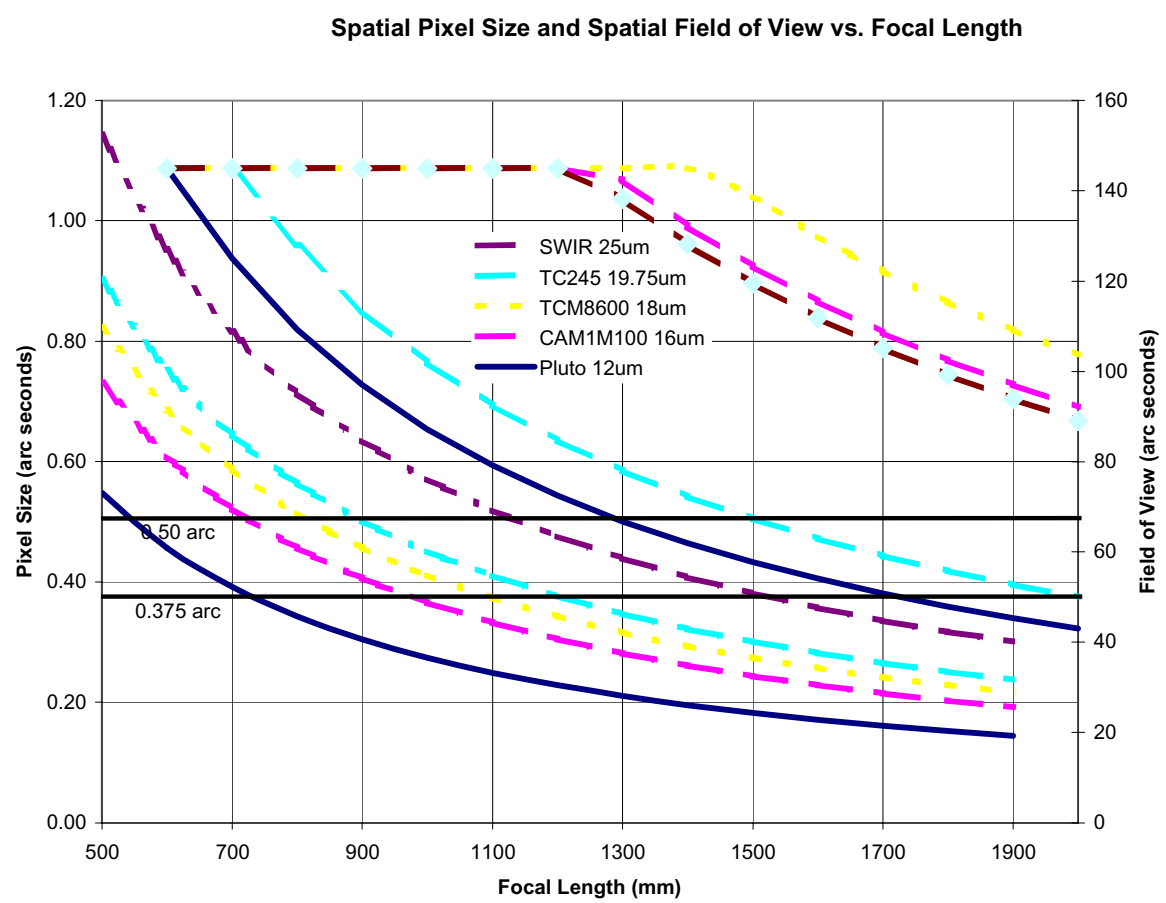

Figure 3. Pixel size and field of view for various cameras and camera lenses.The lower set of curves show pixel size according to the scale on the left. The upper curves show field of view as shown on the scale on the right.

is essentially the same procedure that was used for the ASP calibration, which is explained in the paper by Skumanich et al. (1997) and will not be repeated here. The only difference is that the calibration polarizer and retarder are now almost entirely achromatic over the broad wavelength range of SPINOR. The retardance of the calibration retarder and the orientation of its fast axis are taken as free parameters of the fit, in order to account for wavelength variations and possible mounting inaccuracies. The following are $\mathbf{X}$-matrices measured in the May 2005 run at 587.6, 1083.0 and $1565.0 \mathrm{~nm}$ : 


$$
\begin{aligned}
& \mathbf{X}_{587.6}=\left(\begin{array}{cccc}
1.00 & 0.24 & 4.08 \times 10^{-4} & -6.16 \times 10^{-2} \\
1.79 \times 10^{-2} & 0.46 & 0.15 & 2.99 \times 10^{-2} \\
-4.11 \times 10^{-3} & -0.14 & 0.44 & 0.13 \\
1.84 \times 10^{-3} & 3.41 \times 10^{-2} & 0.15 & -0.46
\end{array}\right)(2) \\
& \mathbf{X}_{1083.0}=\left(\begin{array}{cccc}
1.00 & 0.23 & 4.90 \times 10^{-2} & -6.99 \times 10^{-2} \\
1.17 \times 10^{-2} & 0.45 & 0.18 & 2.47 \times 10^{-2} \\
-3.64 \times^{-2} & -0.18 & 0.45 & 8.19 \times 10^{-2} \\
1.69 \times 10^{-2} & 4.87 \times 10^{-2} & 9.91 \times 10^{-2} & -0.41
\end{array}\right)(3) \\
& \mathbf{X}_{1565.0}=\left(\begin{array}{cccc}
1.00 & 6.22 \times 10^{-2} & 2.95 \times 10^{-4} & -1.38 \times 10^{-2} \\
6.29 \times 10^{-3} & 0.43 & -0.41 & -4.99 \times 10^{-2} \\
-4.25 \times 10^{-4} & 0.44 & 0.43 & 4.08 \times 10^{-2} \\
5.02 \times 10^{-2} & 3.84 \times 10^{-3} & 2.59 \times 10^{-2} & -0.19
\end{array}\right)(4)
\end{aligned}
$$

The telescope calibration requires an entire day of observations at different positions of the Sun on the sky. This needs to be done at least every time the turret mirrors are recoated, and desirably every few months. We have devised a new method that augments that of Skumanich et al. (1997). In that paper, the telescope is modeled as a function of the following free parameters: the entrance window retardance and axis orientation; the turret mirrors retardance and ratio of reflectivies $(r s / r p)$; the main mirror retardance and $r s / r p$; the exit window retardance and axis orientation; and finally the relative rotation between the telescope and the polarimeter (see Skumanich et al. 1997 for an explanation of these parameters).

An array of achromatic linear polarizers, placed in front of the entrance window, is used to introduce known states of polarization in the system. These vectors are measured at various times of the day, covering a wide range of the three variable angles of the telescope: turret azimuth, elevation and table rotation. Again, a least-squares fit is used to determine the free parameters of the model.

The new SPINOR technique uses a cross-dispersing prism placed before one of the detectors to record the various overlapping orders simultaneously (shown in Fig 4). In this manner we are able to obtain telescope calibration data for many wavelengths at once. Instead of fitting each set of parameters independently for each wavelength, we take a fully-consistent approach whereby the entire dataset is fit using a wavelength-dependent model. Some parameters (window retardances and mirror properties) are allowed to vary with wavelength in the fit, up to the third order in $\lambda$. This is enough to account for the non-monotonic 


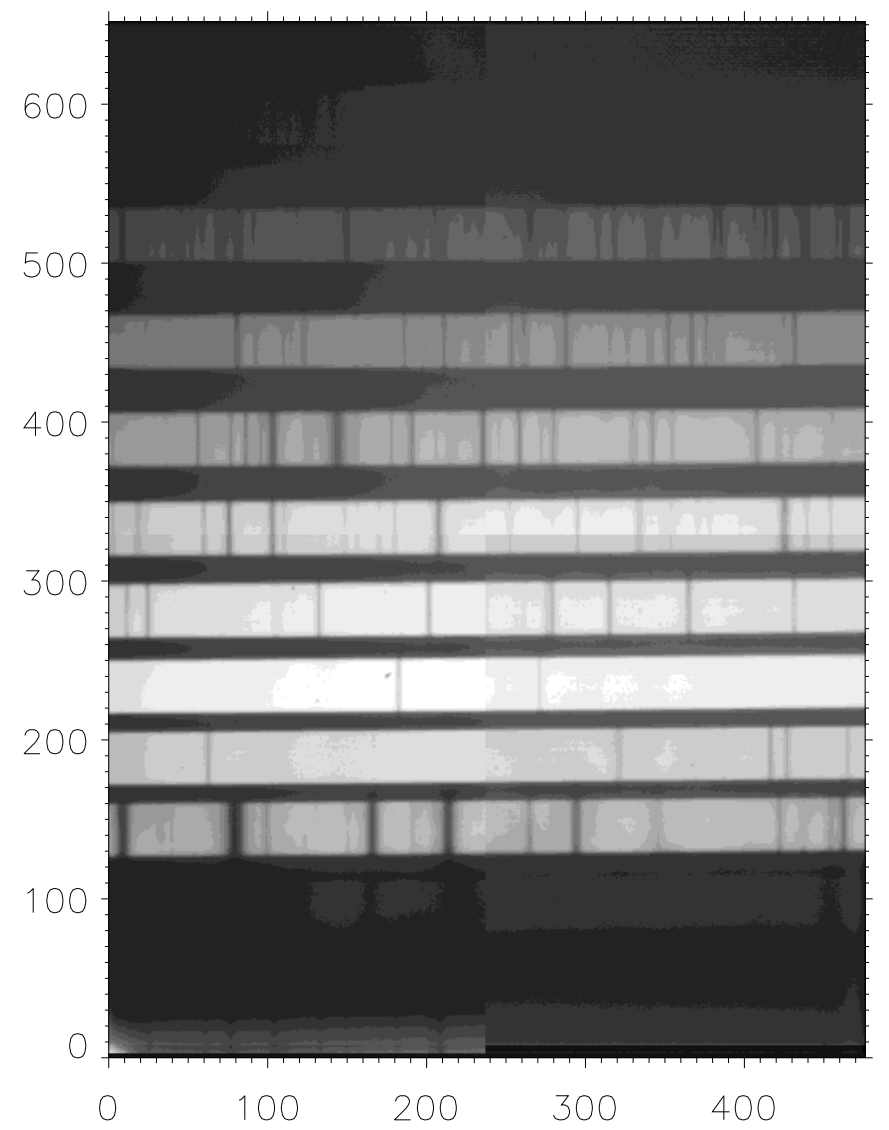

Figure 4. Cross-dispersed spectra from a telescope calibration operation. Central wavelengths in nm from top to bottom: 416, 451, 492, 541, 601, 677, 773, 902.

behavior in the complex refractive index of aluminum in the near infrared. The rest of the parameters are wavelength-independent. This model has sufficient freedom to reproduce the measured vectors in the entire dataset and at the same time provides a consistent model of the telescope. Table III shows the various telescope parameters obtained from our calibration at three different wavelengths. Fig 3.4 shows the wavelength dependence of the retardance and $r_{s} / r_{p}$ of the telescope mirrors and windows. This calibration has been successfully applied on actual data at 587.6, 630.2, 849.8, 854.2, 866.2, 1083.0 and $1565.0 \mathrm{~nm}$, which demonstrates the robustness of the method. 

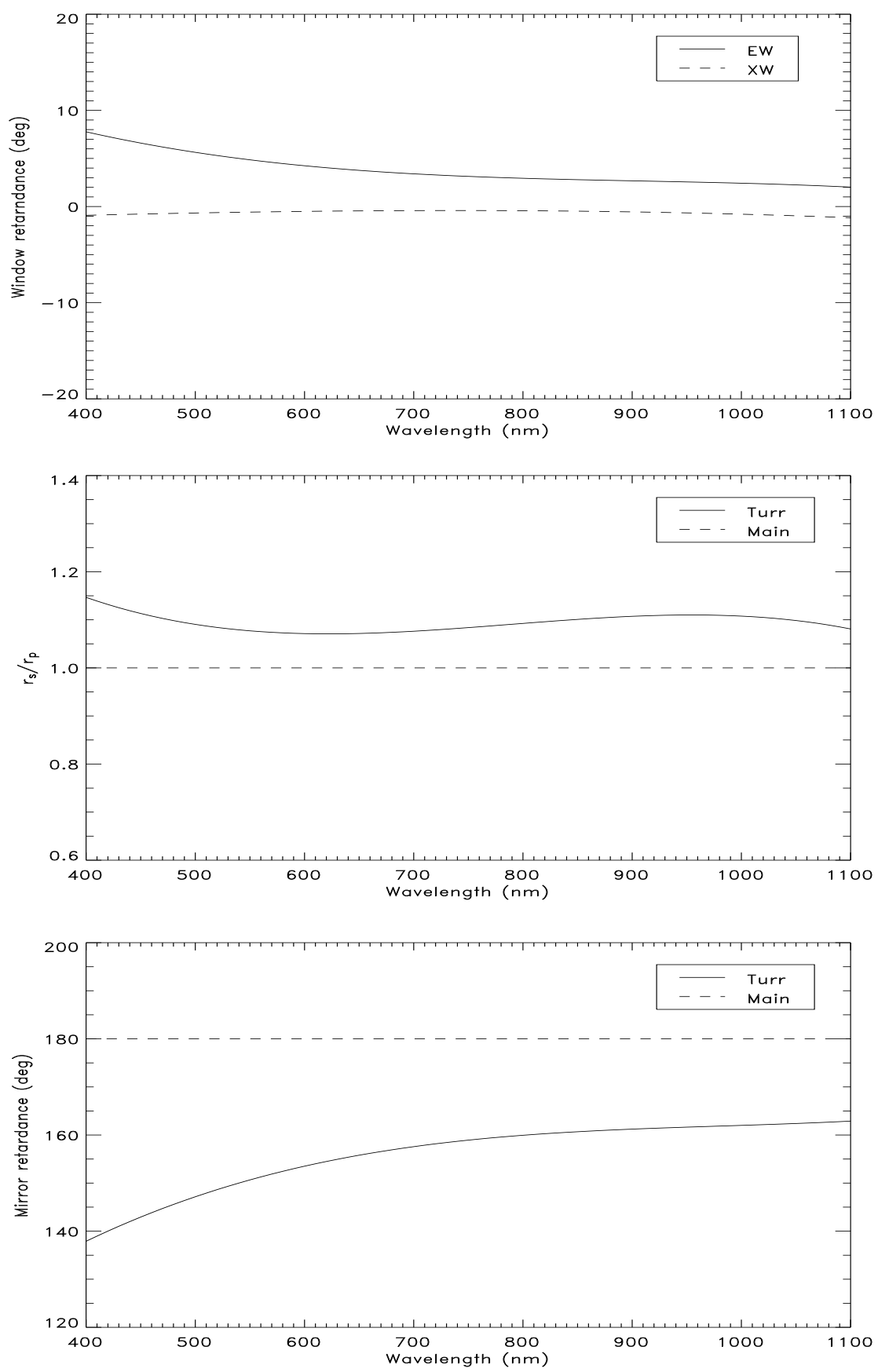

Figure 5. Curves of retardance (degrees) and $r_{s} / r_{p}$ as a function of wavelength, determined from the telescope calibration operation using the cross-dispersed spectra. 
Table III. DST telescope parameters retrieved with our calibration procedure. EW: Entrance window, Turr: turret mirrors, XW: Exit window. All angles are degrees.

\begin{tabular}{lccc}
\hline Wavelength $(\mathrm{nm})$ & 400 & 600 & 800 \\
\hline EW retardance & 7.53 & 3.79 & 2.67 \\
EW orientation & 38.64 & 38.64 & 38.64 \\
Turr. rs/rp & 1.13 & 1.07 & 1.10 \\
Turr. retardance & 139.11 & 155.62 & 161.23 \\
Main rs/rp & 1.00 & 1.00 & 1.00 \\
Main retardance & 179.99 & 179.99 & 179.99 \\
XW retardance & 1.56 & 0.97 & 0.28 \\
XW orientation & 345.81 & 345.81 & 345.81 \\
T-X rotation & 81.26 & 81.26 & 81.26 \\
\hline
\end{tabular}

\section{Observations}

A first engineering run took place on June 2004 with the following configuration. The new polarization and imaging optics were carefully set up and aligned. For much of this run we used the old ASP modulator because at the time of the observations we had not yet received the new achromatic modulator from the manufacturer. This resulted in less than optimum polarimetric efficiency at long wavelengths. The new modulator arrived later in the observing run and is now available for use at the DST. The ASP cameras were configured to record the Ca II lines at 849.8 and $854.2 \mathrm{~nm}$ (plus some other photospheric and telluric lines blended in the wings of the Ca lines). The new Pluto camera was observing at $1083.0 \mathrm{~nm}$, including the He I multiplet, two photospheric lines and a telluric line. We aligned the camera chips so that the broad $\mathrm{Ca}$ lines would fall near the edge of the chip. In this manner we can record some wing "continuum" on the opposite side of the detector, which is useful for the data analysis (polarization calibration, flat-fielding and also as a reference for the thermodynamics in the inversions). Since most of the polarization signal is concentrated near the line core, it is not necessary to record both wings of the Ca lines. An additional advantage is that we can also record a strong photospheric line in the $854.2 \mathrm{~nm}$ images.

Fig 6 shows several maps of active region NOAA 0634 observed on June 16 at 15:16 UT. This dataset has the best seeing among our active region observations, with a granulation contrast of $3.3 \%$ (notice that 
this value may not be directly comparable to that of visible observations because of the variation of the source function with wavelength). Some features apparent in the magnetograms or the chromospheric filtegrams (e.g., the transversal fluctuations in the penumbral filaments near coordinates $[x=50, y=55]$ in the figure) exhibit spatial scales as small as $0.6 "$ ".

The maps represented in the figures have been obtained from a spectro-polarimetric scan of the region consisting of 350 steps of 0.22 " each. The scanning step oversamples the resolution element. This was done so that it would be possible to bin the $1083.0 \mathrm{~nm}$ data in order to build up its photon count without saturating the other two detectors. Unfortunately the $1083 \mathrm{~nm}$ data from this day was unusable due to problems with the camera. Therefore we only show the 849.8 and $854.2 \mathrm{~nm}$ regions for this dataset.

The term "magnetogram" is used in this work somewhat inappropriately (but following a very widespread terminology) to refer to the amount of Stokes V signal integrated over a certain bandwidth on either the red or blue side of the line center, and normalized to the intensity integrated over the same bandwidth. We have used a 10-pixel wide square filter function. Notice that this quantity is not necessarily related to the magnetic field strength in the Sun. In some cases it may bear some resemblance to the longitudinal magnetic flux density (as defined by Graham et al. 2002), but this is not always the case (Socas-Navarro 2002) especially given the complex polarization patterns exhibitted by the chromospheric lines (see Fig 7). For this reason, the red and blue magnetograms represented in Fig 6 are different.

A sample of the slit spectra recorded by SPINOR is presented in Fig 7. The data in the figure are from a scan of NOAA 0635 observed on June 19 at 14:08 UT. The problems with the Pluto camera computer had been solved during the previous days, allowing us to record data at $1083 \mathrm{~nm}$. The signal-to-noise ratio is quite poor due to 1) the low polarimetric efficiency discussed above, and 2) especially to the low quantum efficiency of the camera at this wavelength $(\simeq 3 \%)$ (these issues were addressed in the subsequent May 2005 run by using the new achromatic modulator and the Rockwell detector). The $1083 \mathrm{~nm}$ data displayed in the figure have been binned in the scanning direction over a 1.5" interval. The 849.8 and $854.2 \mathrm{~nm}$ data are unbinned (0.22").

A second observing campaign was recently conducted on May 2005, intended to characterize and demonstrate the capabilities of SPINOR beyond $1 \mu \mathrm{m}$ with the infrared Rockwell camera. In this campaign we carried out observations up to $1565 \mathrm{~nm}$. A sample of such observations after our preliminary analysis is presented in Fig 8. 

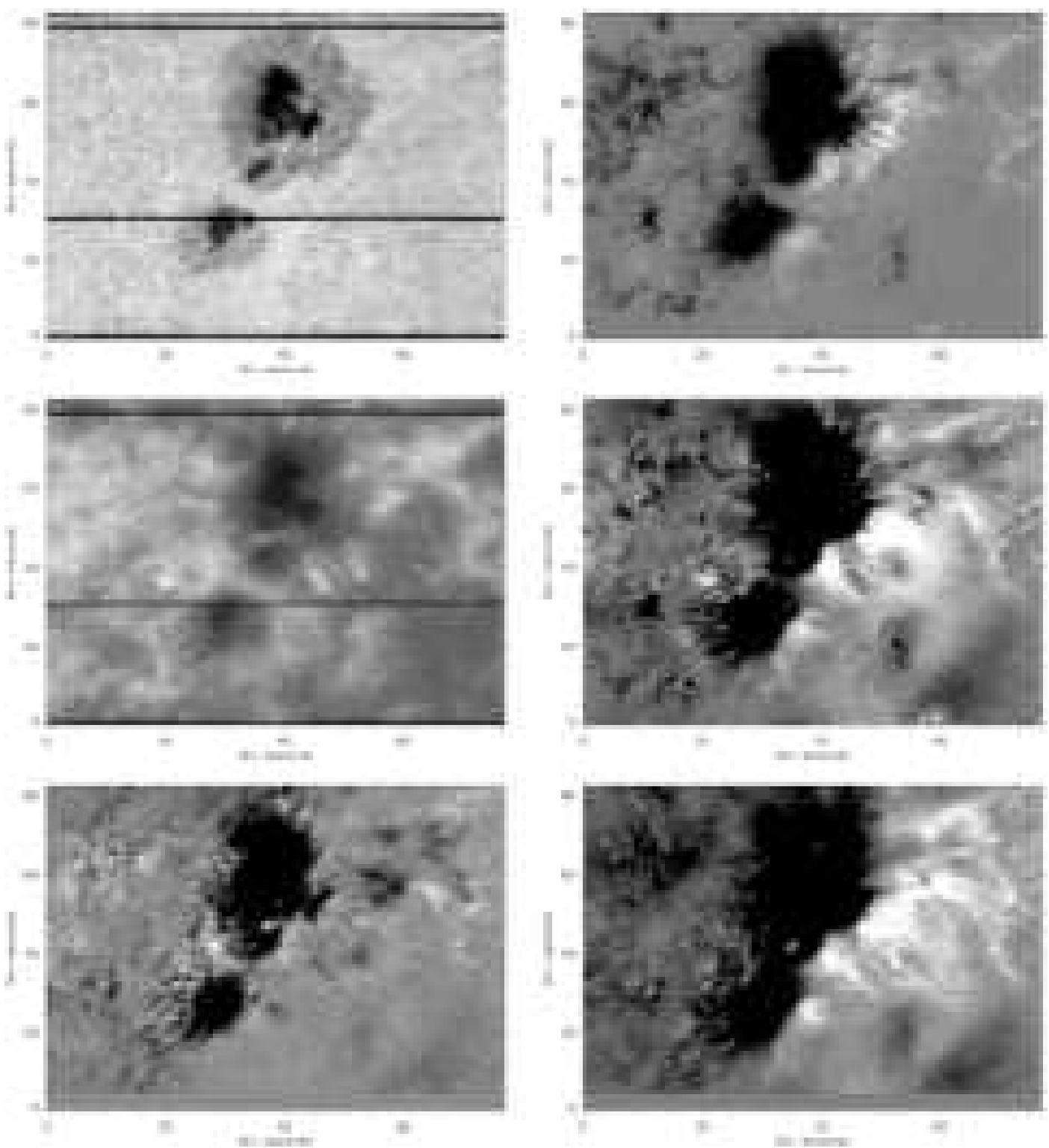

Figure 6. Various maps of active region NOAA 0634. From top to bottom, left to right: Continuum intensity at $854 \mathrm{~nm}$. Photospheric magnetogram. Chromospheric filtergram in the core of the Ca II $854.2 \mathrm{~nm}$ line. Chromospheric magnetogram taken on the red side of the $849.8 \mathrm{~nm}$ line core. Chromospheric magnetogram on the blue side of $854.2 \mathrm{~nm}$. Chromospheric magnetogram on the red side of $854.2 \mathrm{~nm}$. The gray scale in all chromospheric [photospheric] magnetograms is $\pm 2 \%$ [ $\pm 3 \%$ ] of the corresponding Stokes I intensity (see text). Disk center is towards the left of the image. 

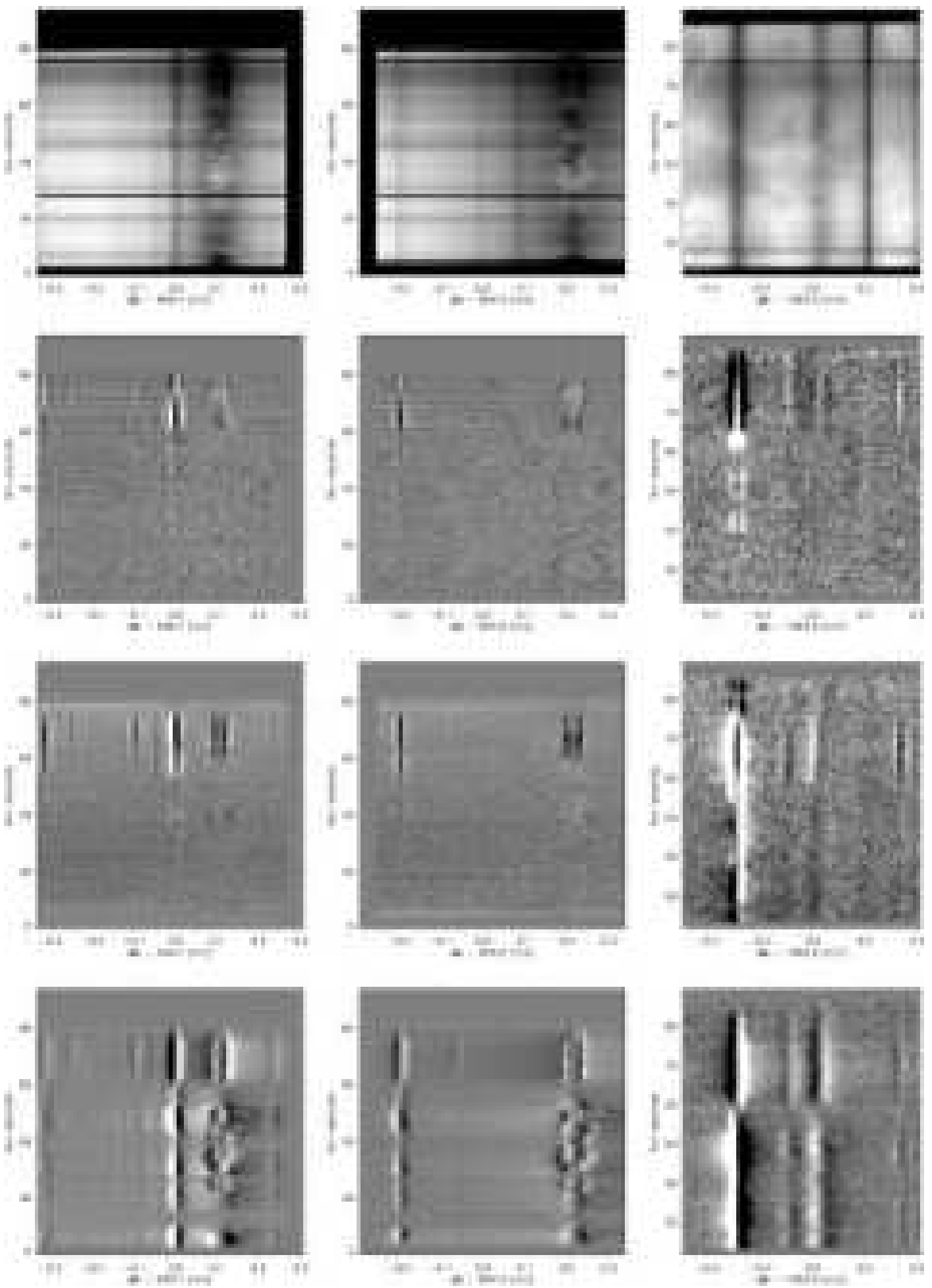

Figure 7. Stokes profiles from one particular slit position of the NOAA 0635 scan. Rows: Stokes I, Q, U and V parameters (from top to bottom). Columns: Images from the camaras at 849.8 (left), 854.2 (middle) and 1083 (right) nm. Profiles are normalized to quiet Sun continuum. Stokes Q and U panels are saturated at $\pm 1 \%$, Stokes V panels are saturated at $\pm 2 \%$. 

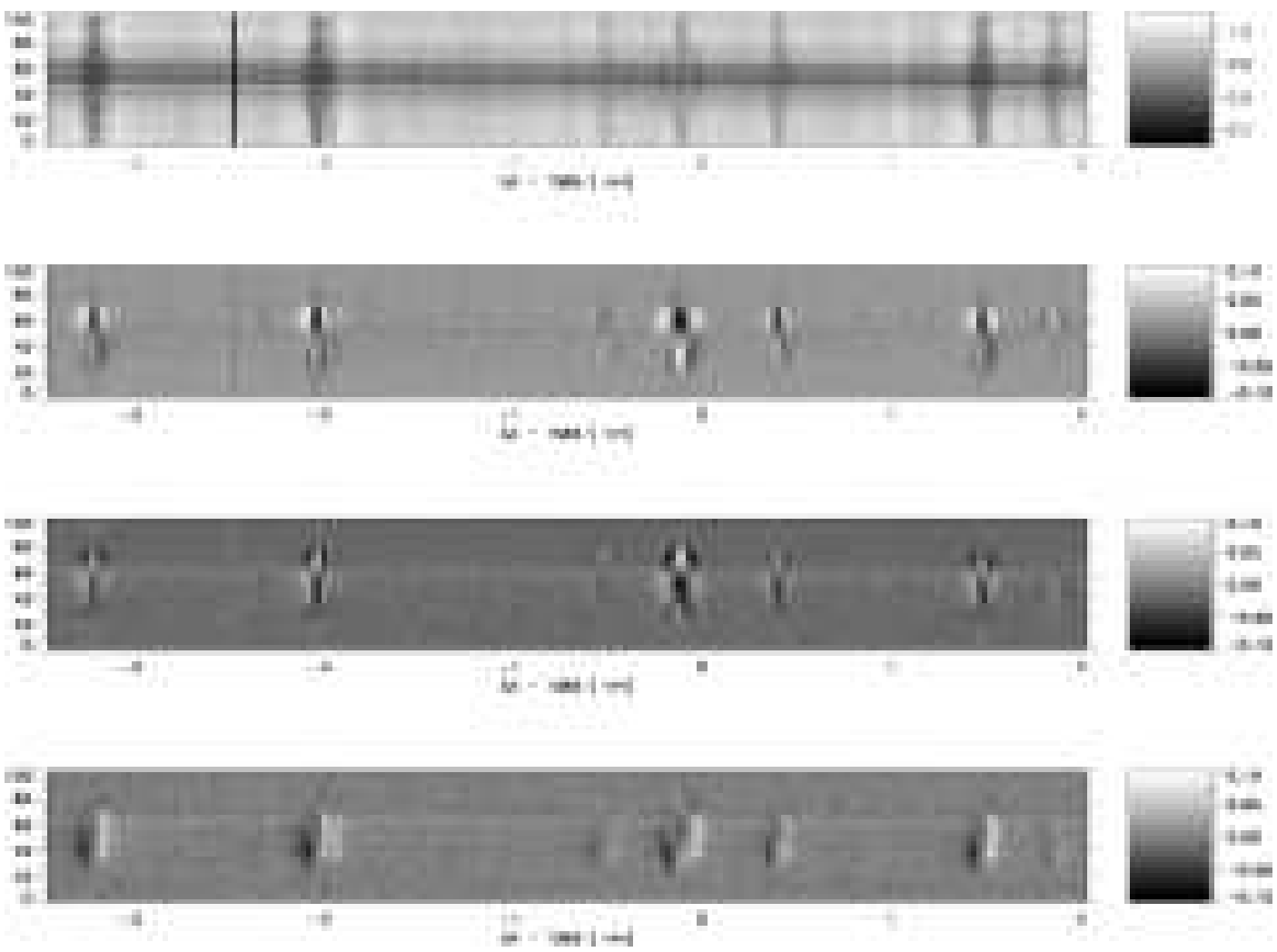

Figure 8. Stokes profiles from one particular slit position of a sunspot map observed on 23 May 2005. Rows: Stokes I, Q, U and V parameters (from top to bottom). Profiles are normalized to quiet Sun continuum. Wavelengths are in nm from 1565.

\section{Conclusions}

This paper presents a detailed description and some initial results from first-light observations with SPINOR, a new facility instrument for the DST. We perceive an urgent demand in the solar community for a new experiment-oriented (as opposed to specialized) spectro-polarimeter. We expect that SPINOR will fulfill this need and remain at the cuttingedge of solar research at least until the construction of the Advanced Technology Solar Telescope (Keller et al. 2002). Its broad wavelength coverage will provide a uniquely connected view of photospheric and chromospheric magnetism. The observations presented in the present paper are intended to emphasize this point and to demonstrate the capabilities of the instrument in the near infrared.

Future work will focus on the implementation of a new control system. SPINOR is currently being operated from the ASP control computer, which sends strobe signals to both ASP cameras and up 
to four other "external" detectors. The new control system, which is expected to be implemented by the end of 2006, will be more userfriendly and take advantage of the virtual camera interface currently under development by NSO for the DST.

As with the ASP, researchers from national and foreign institutions will be able to access the new instrument through the usual time allocation competition for the Dunn Solar Telescope, operated by NSO.

\section{Acknowledgments}

The authors wish to acknowledge the enthusiastic support from the NSO staff at the Sacramento Peak observatory, especially D. Gilliam, M. Bradford and J. Elrod. Thanks are also due to S. Gregory, R. Dunbar, T. Spence, S. Fletcher, C. Berst and W. Jones.

\section{References}

Casini, R. and A. López Ariste: 2003, ". In: Solar Polarization, ASP Conference Series, held Sep 3rd-Oct 4th 2002 at Puerto de la Cruz, Tenerife, Spain. Edited by J. Trujillo Bueno and J. Sánchez Almeida. San Francisco: Astronomical Society of the Pacific, 2003. p. in press.

del Toro Iniesta, J. C., L. R. Bellot Rubio, and M. Collados: 2001, 'Cold, Supersonic Evershed Downflows in a Sunspot'. ApJL 549, L139-L142.

Elmore, D. F., B. W. Lites, S. Tomczyk, A. Skumanich, R. B. Dunn, , J. A. Schuenke, K. V. Streander, T. W. Leach, C. W. Chambellan, Hull, and L. B. Lacey: 1992. In: Proc SPIE, Vol. 1746. p. 22.

Graham, J. D., A. López Ariste, H. Socas-Navarro, and S. Tomczyk: 2002, 'Inference of Solar Magnetic Field Parameters from Data with Limited Wavelength Sampling'. Solar Physics 208, 211-232.

Guimond, S. and D. Elmore: 2004, 'Designing effective crystal waveplates requires understanding the engineering tradeoffs'. SPIE's OE Magazine May 2004, pp 26-29.

Keller, C. U.: 1998, 'SOLIS: a modern facility for synoptic solar observations'. Proc. SPIE 3352, 732-741.

Keller, C. U., T. R. Rimmele, F. Hill, S. L. Keil, J. M. Oschmann, and the ATST Team: 2002, 'The Advanced Technology Solar Telescope'. Astronomische Nachrichten 323, 294-298.

Khomenko, E. V., M. Collados, S. K. Solanki, A. Lagg, and J. Trujillo Bueno: 2003, 'Quiet-Sun inter-network magnetic fields observed in the infrared'. $A \& A 408$, 1115-1135.

López Ariste, A., S. Tomczyk, and R. Casini: 2002, 'Hyperfine Structure as a Diagnostic of Solar Magnetic Fields'. ApJ 580, 519-527.

Lin, H.: 1995, 'On the Distribution of the Solar Magnetic Fields'. ApJ 446, 421.

Lin, H. and T. Rimmele: 1999, 'The Granular Magnetic Fields of the Quiet Sun'. ApJ 514, 448-455. 
Lites, B. W., D. F. Elmore, and K. V. Streander: 2001, "The Solar-B SpectroPolarimeter"'. In: M. Sigwarth (ed.): "Advanced Solar Polarimetry - Theory, Observation, and Instrumentation", Vol. 236 of ASP Conf. Ser. p. 33.

Manso Sainz, R. and J. Trujillo Bueno: 2001, 'Modeling the Scattering Line Polarization of the Ca II Infrared Triplet'. In: ASP Conf. Ser. 236: Advanced Solar Polarimetry - Theory, Observation, and Instrumentation. p. 213.

Martínez Pillet, V., B. W. Lites, A. Skumanich, and D. Degenhardt: 1994, 'Evidence for supersonic downflows in the photosphere of a delta sunspot'. ApJL 425, L113-L115.

Martínez Pillet, V.: 2000, 'Spectral signature of uncombed penumbral magnetic fields'. A\&A 361, 734-742.

Martínez Pillet, V.: 2001, 'Spectral signature of uncombed penumbral magnetic fields. Reply'. A\&A 369, 644-645.

Rimmele, T. R.: 2000, 'Solar adaptive optics'. In: Proc. SPIE Vol. 4007, p. 218-231, Adaptive Optical Systems Technology, Peter L. Wizinowich; Ed. pp. 218-231.

Sánchez Almeida, J.: 2001, 'Spectral signature of uncombed penumbral magnetic fields. Comment'. A $\mathscr{G} A$ 369, 643.

Sánchez Almeida, J. and J. A. Bonet: 1998, 'The Spectrum of Fluctuations across Penumbral Filaments'. ApJ 505, 1010-1017.

Sankarasubramanian, K., D. F. Elmore, B. W. Lites, M. Sigwarth, T. R. Rimmele, S. L. Hegwer, S. Gregory, K. V. Streander, L. M. Wilkins, K. Richards, and C. Berst: 2003, 'Diffraction limited spectro-polarimeter - Phase I'. In: Polarimetry in Astronomy. Edited by Silvano Fineschi . Proceedings of the SPIE, Volume 4843, pp. 414-424 (2003). pp. 414-424.

Schlichenmaier, R.: 2002, 'Penumbral fine structure: Theoretical understanding'. Astronomische Nachrichten 323, 303-308.

Schmidt, W., C. Beck, T. Kentischer, D. Elmore, and B. Lites: 2003, 'POLIS: A spectropolarimeter for the VTT and for GREGOR'. Astronomische Nachrichten 324, 300-301.

Skumanich, A., B. W. Lites, V. Martínez Pillet, and P. Seagraves: 1997, 'The Calibration of the Advanced Stokes Polarimeter'. ApJS 110, 357.

Socas-Navarro, H.: 2002, 'Zeeman diagnostics of solar magnetic fields'. In: SOLMAG 2002. Proceedings of the Magnetic Coupling of the Solar Atmosphere Euroconference and IAU Colloquium 188, 11 - 15 June 2002, Santorini, Greece. Ed. H. Sawaya-Lacoste. ESA SP-505. Noordwijk, Netherlands: ESA Publications Division, ISBN 92-9092-815-8, 2002, p. 45 - 51. p. 45.

Socas-Navarro, H.: 2003, 'Small-scale Magnetic Fields in the Quiet Sun'. In: Solar Polarization, ASP Conference Series, held Sep 3rd-Oct 4th 2002 at Puerto de la Cruz, Tenerife, Spain. Edited by J. Trujillo Bueno and J. Sánchez Almeida. San Francisco: Astronomical Society of the Pacific, Vol 30\%. p. 330.

Socas-Navarro, H., B. Ruiz Cobo, and J. Trujillo Bueno: 1998, "'Non-LTE Inversion of Line Profiles"'. ApJ 507, 470-481.

Socas-Navarro, H. and J. Sánchez Almeida: 2002, 'Magnetic Properties of Photospheric Regions with Very Low Magnetic Flux'. ApJ 565, 1323-1334.

Socas-Navarro, H. and J. Sánchez Almeida: 2003, 'Magnetic Fields in the Quiet Sun: Observational Discrepancies and Unresolved Structure'. ApJ 593, 581.

Socas-Navarro, H., J. Trujillo Bueno, and B. Ruiz Cobo: 2000a, "Anomalous Circular Polarization Profiles in Sunspot Chromospheres"'. ApJ 544, 1141-1154.

Socas-Navarro, H., J. Trujillo Bueno, and B. Ruiz Cobo: 2000b, 'Anomalous Polarization Profiles in Sunspots: Possible Origin of Umbral Flashes'. Science 288, 1396-1398. 
Socas-Navarro, H., J. Trujillo Bueno, and B. Ruiz Cobo: 2000c, "Non-LTE Inversion of Stokes Profiles Induced by the Zeeman Effect"'. ApJ 530, 977-993.

Socas-Navarro, H., J. Trujillo Bueno, and B. Ruiz Cobo: 2001, 'A Time-dependent Semiempirical Model of the Chromospheric Umbral Oscillation'. ApJ 550, 11021112 .

Solanki, S. K., W. Curdt, A. Gandorfer, M. Schüssler, B. W. Lites, V. Martinez Pillet, W. Schmidt, A. M. Title, and The Sunrise Team: 2003, 'SUNRISE: Balloonborne High-Resolution Observation of the Sun'. Astronomische Nachrichten Supplement 324, 113-+.

Uitenbroek, H.: 1989, 'Operator perturbation method for multi-level line transfer with partial redistribution'. A\&A 213, 360-370. 